\title{
Longitudinal Health Care Cost in Hypoplastic Left Heart Syndrome Palliation
}

\author{
Jesse E. Hansen ${ }^{1} \cdot$ Nicolas L. Madsen ${ }^{1} \cdot$ Laurie Bishop ${ }^{2} \cdot$ David L. S. Morales $^{1} \cdot$ Jeffrey B. Anderson ${ }^{1}$
}

Received: 7 February 2018 / Accepted: 2 May 2018 / Published online: 17 May 2018

○) Springer Science+Business Media, LLC, part of Springer Nature 2018

\begin{abstract}
Management of hypoplastic left heart syndrome (HLHS) is resource intensive. Heath care systems are pressured to provide value to patients by improving outcomes while decreasing costs. A single-center retrospective cohort of infants with HLHS who underwent Norwood procedure or hybrid Norwood from 2004 to 2014 and survived to first outpatient follow up were studied. The primary outcome was total cost through 12 months with a sub-analysis of patients with 60 months of data. Costs were calculated using internal cost accounting system and reported by cost center. Of the 152 HLHS patients identified, 69 met inclusion criteria. Stage I hospitalization $(n=69)$, with a median length of stay 34 days [interquartile range (IQR) 24-58 days], resulted in a median cost of $\$ 203,817$ (IQR $\$ 136,236-272,453)$. Of survivors at 12 months $(n=55)$, the median cost was $\$ 369,393$ (IQR $\$ 216,289-594,038$ ) generated in part by a median of 67 (40-126 days) hospitalized days during that year. A subgroup analysis of patients who reached 60 months of age $(n=29)$ demonstrated a median total cost of $\$ 391,812$ (IQR $\$ 293,801-577,443$ ) and a median of 74 lifetime hospitalized days (IQR 58-116 days). High cost centers included intensive care (41\%), non-ICU hospital (17\%), operative services (11\%), catheterization lab (9\%), and pharmacy (9\%). Using multiple regression analysis, significant drivers of cost included reoperation, length of hospitalization, low birthweight, and use of ECMO. Costs related to HLHS management are driven both by care-related complications such as surgical re-intervention and patient factors such as low birth weight.
\end{abstract}

Keywords Cost $\cdot$ Value $\cdot$ Hypoplastic left heart syndrome $\cdot$ Congenital heart disease $\cdot$ Norwood procedure

\section{Introduction}

Congenital heart defects (CHD) occur in approximately 1 out of 100 live births [1] and represent the most common cause of infant death related to birth defects. CHD accounts for $28 \%$ of deaths due to birth defects in the first month of life and approximately $50 \%$ of the deaths due to birth defects during the first 2-12 months [2]. Fortunately, from 1999 to 2014 , death rates attributable to CHD decreased from 1.6 to 0.9 per 100,000 children. Mortality for children who undergo cardiac surgery has decreased to 3.3\% [2]. All the

Jeffrey B. Anderson

Jeffrey.Anderson@cchmc.org

1 Department of Pediatrics, Heart Institute, Cincinnati Children's Hospital Medical Center, 3333 Burnet Avenue, ML2003, Cincinnati, OH 45229, USA

2 Division of Biostatistics and Epidemiology, Department of Pediatrics, Cincinnati Children's Hospital Medical Center, Cincinnati, $\mathrm{OH}$, USA while, hospital costs for pediatric patients with CHD have grown, exceeding $\$ 6.6$ billion in 2012, accounting for $23 \%$ of the total cost for all hospitalizations in children and adolescents aged 0-20 years [2].

Prior studies have focused on various aspects of the cost of care for children born with significant heart defects that require surgery in the first year of life [3-7]. The majority of these studies have used administrative databases to track increasing resource utilization trends [7], wide variation in costs [5], and associations between cost, quality, and medical center volume [4]. The results of these studies have been limited by the structure of the data sets making it difficult to link separate episodes of care for one patient over time. This in turn restricts a longer-term view of the total costs of care. Meaningful studies looking at total cost of care over the first 5 years of life, inclusive of all care episodes both ambulatory and hospital based, have not been published to this date. This concept of understanding costs contributing to longitudinal care is particularly important as we move toward providing value-based care. As outlined by Porter, 
real value in healthcare must be considered over the course of time, rather than relying wholly on episode-based analysis, both in determining costs and outcomes [8]. Previously published work has not addressed all three tiers of Porter's health outcomes hierarchy that may provide further insight into the relative value of care provided [8].

This study aims to more fully define the cost of caring for infants with hypoplastic left heart syndrome (HLHS) surviving the first year of life and to begin examining the cost of care up to the fifth year of life.

\section{Materials and Methods}

\section{Design}

This is a single-center retrospective cohort study of all infants undergoing single-ventricle palliation at Cincinnati Children's Hospital Medical Center from January 2004 to January 2014. Patients were identified using the Society for Thoracic Surgeons data registry to select infants who underwent initial surgical palliation with a Norwood procedure or hybrid Norwood procedure. Inclusion criteria required planned transition to Glenn and/or Fontan physiology and survival to the first outpatient visit. Patients who did not survive the initial hospitalization were excluded from analysis. Infants who had ambulatory care at an outside center were excluded from analysis to isolate those patients with complete financial records. The primary clinical outcome is transplant-free survival, and the primary financial outcome is total cost incurred during the first year of life with a subgroup analysis of total cost of care for the group who had reached their fifth birthday at the time of analysis. Secondary financial analysis included the clinical cost centers that contributed most to the cost of care for these infants during their first year of life.

\section{Measurement}

Demographic data including gestational age at birth, birth weight, sex, and residency geographic location at the level of census tract and census tract data including distance from hospital, median household income, and percent below poverty level were collected for each patient. Due to the heterogeneous population of patients undergoing Norwood procedure, we collected clinical information including anatomic diagnosis and presence or absence of the following clinical factors: any genetic syndrome, coronary anomalies, multiple gestation pregnancy, post-operative complication(s) requiring unplanned surgical intervention, ECMO use at any time, and ECMO use categorized by surgical stage. Aggregated total hospitalization days and number of outpatient clinic visits were calculated from dates of services provided in billing records. Costs within the first year are reported by surgical stages with Stage 1 including data from transfer from the birth hospital to discharge after the Norwood surgery. Cost data from birth hospitals are not included as our center is not a birth hospital.

Total costs were calculated from Cincinnati Children's Hospital cost accounting reports using the detail trial balance of gross patient revenue and expenses after elimination of cross charges and internally purchased services. Direct costs are allocated by the salary expense of each cost center. Indirect costs are calculated on the cost center's building square footage, equipment deprecation, and number of annual nursing hours. Total costs were calculated by summing the direct and indirect costs. Costs are allocated inside each cost center to the charge code level for both hospital and professional fees using relative value units (RVUs). Professional fee RVU schedules are updated annually using the Center for Medicare and Medicaid Services RVU update. Hospital fee RVU schedules are updated biennially after internal cost studies are completed. Cost centers analyzed in our study include pharmacy, radiology, echocardiography, cardiac catheterization laboratory, intensive care unit, operating room, laboratory, outpatient cardiology, and ambulatory care excluding cardiology. Total reimbursement at 1 and 5 years were determined based on payment dates of service from accounts receivable records that included public insurer, private insurer, and out of pocket payments for both hospital and professional fees. All monetary values were inflation adjusted to 2015 dollars using the Bureau of Labor Statistics Consumer Price Index.

\section{Statistics}

All study participants were analyzed through 12 months of life or death/transplant. A subgroup analysis was completed for all patients with 60 months of transplant-free survival to evaluate longer-term cost trends. Descriptive statistics were calculated and reported as median values with interquartile range included. Clinical predictors of increased costs were identified with single-variable Wilcoxon rank-sum test. We attempted to eliminate covariation of clinical predictors for increased cost through multivariate analysis. All statistical analysis was performed in SPSS and JMP 13.1.0.

\section{Institutional Review}

The authors assert that all procedures contributing to this work comply with the ethical standards of United States Code of Federal Regulations 45 CFR 46 on the protection of human subjects and with the Helsinki Declaration of 1975, as revised in 2008. This study has been approved by the institutional review board at Cincinnati Children's Hospital Medical Center. 


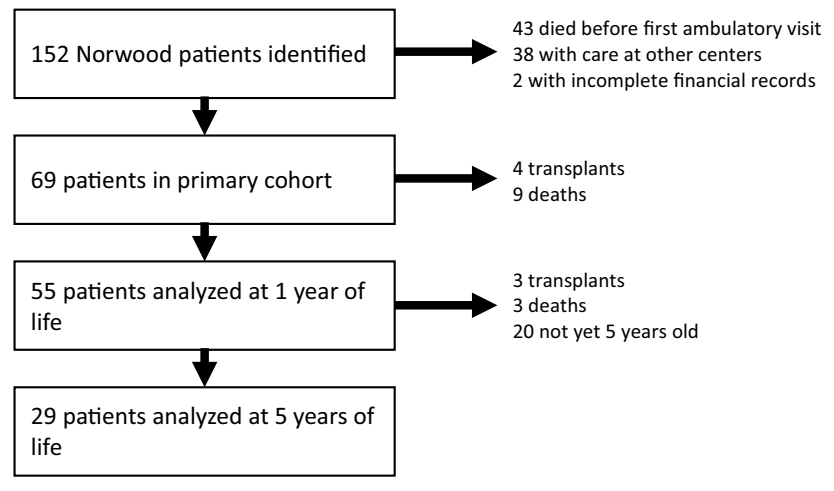

Fig. 1 Cohort size analysis and clinical endpoints

Table 1 Cohort demographics

\begin{tabular}{llll}
\hline & 1 st visit $(N=69)$ & 1 year $(N=55)$ & 5 year $(N=29)$ \\
\hline Sex & 44 males $(64 \%)$ & 35 males $(64 \%)$ & 19 males $(65 \%)$ \\
Gest age & 39 weeks & 39 weeks & 39 weeks \\
Birthweight & $3180 \mathrm{~g}$ & $3180 \mathrm{~g}$ & $3170 \mathrm{~g}$ \\
Race & & & \\
Black & $9(13 \%)$ & $5(9 \%)$ & $3(10 \%)$ \\
White & $60(86 \%)$ & $50(91 \%)$ & $26(90 \%)$ \\
Median income & $\$ 51,135$ & $\$ 52,239$ & $\$ 51,187$ \\
Anatomy & & & \\
HLHS & $46(66 \%)$ & $34(62 \%)$ & $17(48 \%)$ \\
AVC & $8(12 \%)$ & $7(13 \%)$ & $5(17 \%)$ \\
DILV & $4(6 \%)$ & $4(7 \%)$ & $3(10 \%)$ \\
DORV & $5(7 \%)$ & $4(7 \%)$ & $2(7 \%)$ \\
Heterotaxy & $3(4 \%)$ & $3(5 \%)$ & $2(7 \%)$ \\
\hline
\end{tabular}

HLHS hypoplastic left heart syndrome, $A V C$ atrioventricular canal, $D I L V$ double inlet left ventricle, DORV double outlet right ventricle

\section{Results}

Of 152 HLHS patients identified, 71 met inclusion criteria. Two subjects were excluded due to incomplete financial record availability leaving 69 patients for analysis. See Fig. 1 for cohort size analysis. Cohort demographics at each analysis point can be found in Table 1. Cost and length of stay, listed in Table 2, includes all care through discharge for each surgical stage.

The median length of stay for the stage 1 hospitalization was 34 days with an interquartile range (IQR) of 24-58 days. The median cost of stage 1 hospitalization was $\$ 203,817$ with an IQR of $\$ 136,236-272,453$. By 12 months of life, 4 patients had received heart transplants and 9 patients had died leaving 55 patients for analysis. The median days spent hospitalized in the first year of life was 67 with an IQR of 40-126 days including a median of 33 ICU days. The median cost of all care at 12 months was $\$ 329,308$ with an IQR of $\$ 216,289-594,038$. Patients who did not require ECMO $(n=60)$ incurred a median cost per hospital day of \$5411 with an IQR of \$4593-6136. For those who did require ECMO $(n=9)$, median cost per hospital day was $\$ 6204$ with an interquartile range of \$6003-7208 $(p=0.002)$. Insurance coverage was as follows: $60 \%$ had some form of private insurance, $52 \%$ received some form of public insurance, including $12 \%$ of patients that had both private and public insurance. Private payers were responsible for $74 \%$ of total payments.

At 5 years of life, 3 patients had undergone cardiac transplant and 3 patients had died. 20 patients were not yet 5 years of age leaving 29 patients for analysis. The median total cost was $\$ 391,812$ with an IQR of $\$ 293,801-577,443$. Insurance coverage was as follows: $55 \%$ had some form of private insurance, $65 \%$ received some form of public insurance, including $20 \%$ patients that had both private and public insurance. In total, 3 patients (10\%) that did not qualify for public insurance at 1 year received some form of public insurance at 5 years. Private payers were responsible for $65 \%$ of total payments at 5 years.

Resource utilization breakdown for total cost of care by cost center for all 69 patients in the primary cohort is displayed in Table 3 . High cost centers included intensive care (41\%), non-ICU hospital care (17\%), operating room (11\%), cath lab (9\%), and pharmacy (9\%). Medical, anatomic, and socioeconomic risk factors that have been reported as significant in congenital heart disease outcomes were assessed for association with increased cost at 12 months of life and are listed in Table 4. In multivariable analysis, significant drivers of cost at 12 months of age included reoperation during stage 1 or stage 2 , length of hospitalization, low birthweight, and use of ECMO (all $p<0.05$ ).
Table 2 Median length of stay and median total cost

\begin{tabular}{llll}
\hline & 1 st visit $(N=69)$ & 1 year $(N=55)$ & 5 year $(N=29)$ \\
\hline Median LOS & 34 days & 67 days & 74 days \\
Interquartile range & $24-56$ days & $40-126$ days & $58-116$ days \\
Median cost & $\$ 203,817$ & $\$ 369,393$ & $\$ 391,812$ \\
Interquartile range & $\$ 136,236-\$ 272,453$ & $\$ 216,289-\$ 594,038$ & $\$ 293,801-\$ 577,443$ \\
\hline
\end{tabular}

LOS Length of stay 
Table 3 Resource utilization by cost center

\begin{tabular}{ll}
\hline Cost center & \% Total cost \\
\hline Intensive care & 40.9 \\
Non-ICU hospital & 17.0 \\
Operating room & 11.3 \\
Cardiac catheterization & 9.0 \\
Pharmacy & 8.8 \\
Laboratory & 3.1 \\
Echocardiography & 2.8 \\
Radiology & 2.5 \\
Non-cardiac ambulatory care & 2.2 \\
Cardiac ambulatory care & 1.2 \\
Other & 1.2 \\
\hline
\end{tabular}

Table 4 Risk factor association with total cost at 12 months

\begin{tabular}{|c|c|c|}
\hline Risk factor & $\begin{array}{l}\text { Univariate analy- } \\
\text { sis ( } p \text {-value) }\end{array}$ & $\begin{array}{l}\text { Multivari- } \\
\text { ate analysis } \\
\text { ( } p \text {-value) }\end{array}$ \\
\hline \multicolumn{3}{|l|}{ Medical factors } \\
\hline Sex & 0.39 & - \\
\hline Genetic syndrome & 0.95 & - \\
\hline Stage 1 reoperation ${ }^{\mathrm{a}}$ & 0.01 & $<0.001$ \\
\hline Stage 1 STS complications & 0.61 & - \\
\hline Stage 2 reoperation ${ }^{\mathrm{a}}$ & $<0.001$ & 0.003 \\
\hline Stage 2 STS complications ${ }^{\mathrm{a}}$ & $<0.001$ & - \\
\hline Hybrid Norwood procedure ${ }^{\mathrm{a}}$ & 0.03 & - \\
\hline Any ECMO ${ }^{\mathrm{a}}$ & 0.001 & 0.010 \\
\hline Birth weight ${ }^{\mathrm{a}}$ & $<0.001$ & 0.007 \\
\hline \multicolumn{3}{|l|}{ Anatomic factors } \\
\hline Heterotaxy syndrome & 0.77 & - \\
\hline Atrioventricular canal defect ${ }^{\mathrm{a}}$ & 0.05 & - \\
\hline HLHS & 0.19 & - \\
\hline DILV & 0.61 & - \\
\hline DORV & 0.87 & - \\
\hline Coronary anomaly & 0.57 & - \\
\hline \multicolumn{3}{|l|}{ Socioeconomic factors } \\
\hline Non-white race & 0.85 & - \\
\hline Distance from hospital & 0.92 & - \\
\hline Median household income & 0.49 & - \\
\hline Percent below poverty line & 0.36 & - \\
\hline
\end{tabular}

STS Society of Thoracic Surgeons, ECMO extracorporeal membrane oxygenation, HLHS hypoplastic left heart syndrome, DILV double inlet left ventricle, $D O R V$ double outlet right ventricle

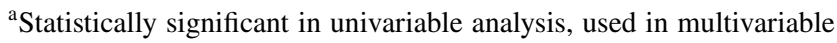
analysis

- Non-significant in multivariable analysis

\section{Discussion}

This study describes the cost of care for hypoplastic left heart syndrome over a period of 12 to 60 months. We emphasize the importance of addressing the longitudinal nature of health care expenditures in medically complex children. Using a cohort cared for at a single center, we tracked patients over time and determined total cost of care as well as where and when their care was most costly. These data provide a first step in targeting longitudinal value-based quality improvement projects in our institution.

Pasquali et al. have previously shown that cost decreases and quality improves as center volume increases and that wide variation exists in costs between referral centers [4-6]. Studies including data obtained from the Pediatric Health Information System database have provided important information about variation in cost of care, but these examinations do not allow for understanding of cost of care over time. In order to begin to tackle the task of providing high-value, long-term care, we need to turn our attention to protensive outcomes as well as cost of care.

This report confirms that care of hypoplastic left heart syndrome is considerable and persistent over years of management. Despite the complicated nature of caring for these infants, this report does provide some potential targets for reducing cost of care. The intensive care unit and surgical complications that result in prolonged ICU stays weigh heavily in the value equation. Continued diligence to improve surgical technique and reduce post-operative complications could have a significant impact on cost of care. Inpatient (non-ICU) costs as well as operating room and cardiac catheterization costs are also important contributors to total cost of care. Future work might focus on how multi-institutional networks like the National Pediatric Cardiology Quality Improvement Collaborative, the Pediatric Critical Care Cardiology Consortium, and the Pediatric Acute Care Cardiology Collaborative can share externally applicable resource utilization and cost information to help identify and implement cost-effective care strategies.

Risk factors for poor CHD outcomes that have been reported in other studies like distance from birth hospital [9], socioeconomic disadvantage [10], and single-ventricle anatomic variants [11] were not found to be significant in our analysis. This discordance with previously published data is likely due to our moderate sample size and the parameters of the inclusion criteria, including selecting only those patients who underwent surgery, survived to discharge, and then remained within the Cincinnati Children's ambulatory cardiology clinic structure. Our study is also limited by the inability to externalize the absolute 
value of our cost numbers to other centers due to differences in cost accounting that exist across different congenital heart centers. It is likely though that the relative resource allocation is similar at other medium-to-high volume surgical centers.

There has been a particular focus on controlling costs and improving quality and safety in health care over the past few decades. These economic and cultural drivers of change in medicine have joined under the common banner of "value" more recently. Value has been broadly defined as increasing quality of care for the same financial cost, or decreasing costs without sacrificing quality. In pediatrics, CHD has been at the center of the value discussion due to the resource intensive nature of care required and life-long implications of improving treatment outcomes. This work will require better understanding of costs through clinically relevant cost accounting methods that allow institutions to identify and promote high-value providers, programs, and quality improvement initiatives.

After cost, the second part of the value equation is quality. Health economists have argued that patient outcomes like mortality, stroke, kidney failure, and health care-associated infections are more valuable to patients than process measures like rates of aspirin adherence, time to first antibiotic administration, and number of patients receiving ventilatorassociated infection prevention bundles that are commonly present in quality improvement work $[8,12,13]$. Our primary clinical outcome was transplant-free survival. While this is a strong clinical endpoint, it is unable to capture the significant morbidity that can be associated with the recurrent and prolonged hospitalizations that children with hypoplastic left heart experience. We are also unable to capture the social and emotional costs to the families, communities, and school districts that bear the responsibility for nurturing our medically complex children. We feel strongly that understanding value in medicine must attempt to include these factors in the development of institutional protocols, society guidelines, and public and private value-based reimbursement programs.

\section{Conclusion}

We have for the first time described the longitudinal cost of care for successful management of hypoplastic left heart through ages 12 and 60 months. In doing so, we have identified several cost centers and patient factors associated with higher costs of care. The aim now becomes translating this new knowledge into cost reduction while maintaining, or improving, the patient outcomes.

Acknowledgements The authors would like to acknowledge the support of the Heart Institute Research Core at Cincinnati Children's
Hospital Medical Center in the development and completion of this project. We also appreciate Dr. Andrew F. Beck's time and expertise in collecting and analyzing the geographic and socioeconomic measures included in our data set.

Funding This research received no specific grant from any funding agency, commercial or not-for-profit sectors.

\section{Compliance with Ethical Standards}

Conflict of interest The authors declare that they have no conflict of interest.

Ethical Approval The authors assert that all procedures contributing to this work comply with the ethical standards of United States Code of Federal Regulations 45 CFR 46 on the protection of human subjects and with the Helsinki Declaration of 1975, as revised in 2008, and has been approved by the institutional review board at Cincinnati Children's Hospital Medical Center.

\section{References}

1. Reller MD, Strickland MJ, Riehle-Colarusso T, Mahle WT, Correa A (2008) Prevalence of congenital heart defects in metropolitan Atlanta, 1998-2005. J Pediatr 153:807-813

2. Benjamin EJ, Blaha MJ, Chiuve SE, Cushman M, Das SR, Deo R, de Ferranti SD, Floyd J, Fornage M, Gillespie C, Isasi CR, Jiménez MC, Jordan LC, Judd SE, Lackland D, Lichtman JH, Lisabeth L, Liu S, Longenecker CT, Mackey RH, Matsushita K, Mozaffarian D, Mussolino ME, Nasir K, Neumar RW, Palaniappan L, Pandey DK, Thiagarajan RR, Reeves MJ, Ritchey M, Rodriguez CJ, Roth GA, Rosamond WD, Sasson C, Towfighi A, Tsao CW, Turner MB, Virani SS, Voeks JH, Willey JZ, Wilkins JT, Wu JH, Alger HM, Wong SS, Muntner P, Subcommittee AHASCaSS (2017) Heart disease and stroke statistics-2017 update: a report from the American Heart Association. Circulation 135:e146-e603

3. Faraoni D, Nasr VG, DiNardo JA (2016) Overall hospital cost estimates in children with congenital heart disease: analysis of the 2012 kid's inpatient database. Pediatr Cardiol 37:37-43

4. Pasquali SK, Sun JL, d'Almada P, Jaquiss RD, Lodge AJ, Miller N, Kemper AR, Lannon CM, Li JS (2011) Center variation in hospital costs for patients undergoing congenital heart surgery. Circ Cardiovasc Qual Outcomes 4:306-312

5. Pasquali SK, Jacobs ML, He X, Shah SS, Peterson ED, Hall M, Gaynor JW, Hill KD, Mayer JE, Jacobs JP, Li JS (2014) Variation in congenital heart surgery costs across hospitals. Pediatrics 133:e553-e560

6. Pasquali SK, Jacobs JP, Bove EL, Gaynor JW, He X, Gaies MG, Hirsch-Romano JC, Mayer JE, Peterson ED, Pinto NM, Shah SS, Hall M, Jacobs ML (2015) Quality-cost relationship in congenital heart surgery. Ann Thorac Surg 100:1416-1421

7. Smith AH, Gay JC, Patel NR (2014) Trends in resource utilization associated with the inpatient treatment of neonatal congenital heart disease. Congenit Heart Dis 9:96-105

8. Porter ME (2010) What is value in health care? N Engl J Med 363:2477-2481

9. Morris SA, Ethen MK, Penny DJ, Canfield MA, Minard CG, Fixler DE, Nembhard WN (2014) Prenatal diagnosis, birth location, surgical center, and neonatal mortality in infants with hypoplastic left heart syndrome. Circulation 129:285-292

10. Kucik JE, Nembhard WN, Donohue P, Devine O, Wang Y, Minkovitz CS, Burke T (2014) Community socioeconomic disadvantage 
and the survival of infants with congenital heart defects. Am J Public Health 346:e150-e157

11. Daebritz SH, Nollert GD, Zurakowski D, Khalil PN, Lang P, del Nido PJ, Mayer JE Jr, Jonas RA (2000) Results of Norwood stage I operation: comparison of hypoplastic left heart syndrome with other malformations. J Thorac Cardiovasc Surg 119:358-367
12. Porter ME, Pabo EA, Lee TH (2013) Redesigning primary care: a strategic vision to improve value by organizing around patients' needs. Health Aff (Millwood) 32:516-525

13. James BC, Poulsen GP (2016) The case for capitation. Harvard Bus Rev 94:102-111 